EPJ manuscript No.

(will be inserted by the editor)

\title{
Effective field theory for spinor dipolar Bose Einstein condensates
}

\author{
M. Takahashi ${ }^{1}$, Sankalpa Ghosh ${ }^{2}$, T. Mizushima ${ }^{1}$, and K. Machida ${ }^{1}$ \\ 1 Department of Physics, Okayama University, Okayama 700-8530, Japan \\ 2 Department of Physics, Indian Institute of Technology, Delhi, Hauz Khas, New Delhi 110016, India \\ Received: date / Revised version: date
}

\begin{abstract}
We show that the effective theory of long wavelength low energy behavior of a dipolar BoseEinstein condensate(BEC) with large dipole moments (treated as a classical spin) can be modeled using an extended Non-linear sigma model (NLSM) like energy functional with an additional non-local term that represents long ranged anisotropic dipole-dipole interaction. Minimizing this effective energy functional we calculate the density and spin-profile of the dipolar Bose-Einstein condensate in the mean-field regime for various trapping geometries. The resulting configurations show strong intertwining between the spin and mass density of the condensate, transfer between spin and orbital angular momentum in the form of Einstein-de Hass effect, and novel topological properties. We have also described the theoretical framework in which the collective excitations around these mean field solutions can be studied and discuss some examples qualitatively.
\end{abstract}

PACS. 03.75.Mn Multicomponent condensates; spinor condensates - 03.75.Hh Static properties of condensates; thermodynamical, statistical, and structural properties - 67.57.Fg Textures and vortices

\section{INTRODUCTION}

$O(3)$ Non-linear sigma model (NLSM) is one of the most studied exactly solvable model in the non-linear field theory. Particularly in two spatial dimension all its finite energy, stable topologically non-trivial solutions are analytically known due to the pioneering work by Belavin and Polyakov [1,2]. In more than two dimensions no such finite energy stable topologically non- trivial solution exists according to a theorem by Derrick and Hobart. However, in spinorial Bose-Einstein condensates, for the relevant time scale for experimental systems, non-trivial texturing can occur in the order parameter field also in three spatial dimensionsn [3] and other type of interaction may actually stabilize them. In real physical systems, effective field theories describing a number of low dimensional condensed matter systems, such as single and multiple layer quantum Hall systems 5, 6, 7, spinorial BECs [8, 9 etc., under various approximations often turn out to be various extensions of NLSM. Of interest to us in this present paper are spinorial BECs, in which all hyperfine states are liberated and the order parameter of the condensates is described by a multi-component vector. This is a fertile ground of the manifestation of the various topological phases of NLSM [8,9]. Even though some of these configurations [3] are actually metastable, their study reveals interesting dynamics related to the internal structures of such spinorial condensates. Spinorial BECs have been realized in fully optically trapped ${ }^{87} \mathrm{Rb}$ and ${ }^{23} \mathrm{Na}$ gases with the hyperfine spin $F=1$ and $F=2$ [10,11] respectively. In all these experiments the dominant interatomic interaction is however short range interactions and is characterized by the corresponding $s$-wave scattering lengths in various spin channels. The other type of interactions, most prominent of which is the dipole-dipole (d-d) interaction among atoms can generally be neglected under most of the current experimental situations. This situation however changed since Bose-Einstein condensates that exhibits such dipole-dipole interaction has recently been achieved by Griesmaier et al. by cooling ${ }^{52} \mathrm{Cr}$ atom gases in a magnetic trap, whose magnetic moment per atom is $6 \mu_{B}$ (Bohr magneton) [12. These experiments are generally performed in presence of a strong magnetic field where all the dipoles are polarized and completely frozen at the typical experimental temperature. Nevertheless, there has been already emerging several novel aspects associated with larger magnetic moment in ${ }^{52} \mathrm{Cr}$ atom gases [13, 14, which is caused by the magnetic dipoledipole (d-d) interaction. Many theoretical studies for such dipolar BECs that have been done [15] are in this limit where the spin degrees of freedom is frozen and one has to deal with a scalar order parameter. In contrast, the theoretical studies of spinor BECs with the d-d interaction are rather limited and few papers exist mostly for the $F=1$ case [16, 17, 18, 19,20]. To study the spinor dipolar BEC with arbitrary value of the spin $F$, one has to handle $2 F+1$ components of spinor and $F+1$ interaction channels. Even for ${ }^{52} \mathrm{Cr}$ case $(F=3)$, we have to solve 7 different equations at the same time in the 4 dimension interaction parameter space 21,22 . This is quite formidable and 
Please give a shorter version with: \authorrunning and \titlerunning prior to \maketitle

complicated. Moreover, interesting topological properties as well as spin-texturing aspects of the resulting ground states configurations are not readily tractable. An alternative approach is to look at the large spin-limit. From the spin-wave analysis of a magnetic systems we know that such analysis works very well when the spin fluctuations is essentially a low energy and long-wavelength process as in the case of large spin. Even for small spin sometimes it works very well. Using this approach, in this paper we model the spinor dipolar Bose-Einstein condensates by treating the spin or the dipole moment of the atom as a freely rotating classical unit vector, namely a classical spin. The resulting theory that represents only the lowenergy long-wavelength behavior of the spin fluctuation in such dipolar condensate is an effective energy functional whose leading terms resemble those of Non-linear sigma model (NLSM) hamiltonian embedded in three spatial dimensions, but accompanied by a non-local term that represents the effect of long-range d-d interaction. Since the original NLSM is rotationally invariant this additional term helps us to understand the effect of symmetry breaking anisotropic long-ranged interaction on the rotationally invariant spin-textures in general. We shall particularly point out some interesting topological features of such solutions, interplay between spin and mass density, interplay between spin and orbital angular momentum.

Some results have been already reported in a recent letter [23. Here we provide a more detailed version of this effective theory and its outcomes by exploring the ground states over a larger region of the parameter space, plot of the resulting ground states and their associated topological properties and how they changed as function of the trap geometry. We additionally provide a theoretical framework in which collective excitations can be studied and a detailed comparison with other methods available to study similar systems.

The rest of the paper is organized as follows. We start with a brief introduction of the non-linear sigma model and its interesting properties in Sec. 2. In the same section we shall the describe the full second quantized hamiltonian for the spinor-dipolar BECs and the resulting effective energy functional in the limit of large dipole moments. Then we shall discuss in Sec. 3 the ground states obtained by minimizing this effective energy functional for various trap geometries. We shall particularly emphasize the ground state for pancake trap and cigar shaped trap, two most widely used geometries. After this Sec. 4 will contain the issue of stability of such condensates and the related quantum phase transitions. In the next section ( Sec. 5) we provide the theoretical framework in which the collective excitations over these phases can be studied. This is followed by a comparison with a set of other works that addressed similar systems (Sec. 6). We shall conclude these discussions by pointing out the experimental implications of our findings and the possibilities of future studies.

\section{NLSM AND THE EFFECTIVE ENERGY FUNCTIONAL FOR SPINOR DIPOLAR CONDENSATE}

We start with a brief description of the prototype nonlinear sigma model and properties of its solutions [1]. The model contains real scalar fields $\phi^{\alpha}(\mathbf{x})$ with $\alpha=1,2,3$ with the following non-linear constraint being imposed at each co-ordinate point $\mathbf{x}$

$$
\phi^{\alpha}(\mathbf{x}) \phi^{\alpha}(\mathbf{x})=\mathbf{1}
$$

The minimal energy functional that is invariant under global $O(3)$ rotation in the internal space of these scalar fields is given by

$$
E=\rho_{s} \int d \mathbf{x}\left(\partial_{\mu} \phi^{\alpha}\right)^{2}
$$

Here $\rho_{s}$ is a constant and $\mu$ refers to the co-ordinate variable. The resulting field equations are non-linear because the above energy functional (2) need to be extremized after implementing the normalization constraint (11). Particularly in two-dimension these equations have stable topologically non-trivial solutions (for a review see ref. [2]). Whereas stable solutions are available in $3 D$ only in presence of an additional gauge interaction [24]. In the dipolar BECs the constant value of the dipole moment of each atom implements the above non-linear constraint (1) and the gradient part of the effective energy functional is manifestly same as the $O(3)$ invariant NLSM model. However the effective energy contains other terms, particularly the anisotropic dipole-dipole interaction, that breaks explicitly this $O(3)$ symmetry of the NLSM. This effective energy functional thus produced upon minimization a different set of solutions depending on the geometry of the trap in which the condensate is confined.

Given these properties we shall now first see what type of approximations gives us this extended NLSM description of the dipolar condensate from the full second quantized Hamiltonian. The full second quantized Hamiltonian that incorporates effect of the dipole-dipole interaction is given as:

$$
\begin{aligned}
H & =\int d \boldsymbol{r}_{i}\left\{\hat{\psi}_{m_{1}}^{\dagger}\left(\boldsymbol{r}_{i}\right)\left(-\frac{\hbar^{2}}{2 m} \nabla^{2}+V_{\text {trap }}\left(\boldsymbol{r}_{i}\right)-\mu\right) \hat{\psi}_{m_{1}}\left(\boldsymbol{r}_{i}\right)\right. \\
& \left.+\sum_{F=0}^{2 f} \mathcal{G}_{F}\left\langle m_{1} m_{2}\left|\mathcal{P}_{F}\right| m_{3} m_{4}\right\rangle \hat{\psi}_{m_{1}}^{\dagger}\left(\boldsymbol{r}_{i}\right) \hat{\psi}_{m_{2}}^{\dagger}\left(\boldsymbol{r}_{i}\right) \hat{\psi}_{m_{3}}\left(\boldsymbol{r}_{i}\right) \hat{\psi}_{m_{4}}\left(\boldsymbol{r}_{i}\right)\right\} \\
& +\frac{c_{d d}}{2} \int d \boldsymbol{r}_{i} \int d \boldsymbol{r}_{j}\left\{\hat{\psi}_{m_{1}}^{\dagger}\left(\boldsymbol{r}_{i}\right) \hat{\psi}_{m_{2}}^{\dagger}\left(\boldsymbol{r}_{j}\right) \hat{\psi}_{m_{3}}\left(\boldsymbol{r}_{j}\right) \hat{\psi}_{m_{4}}\left(\boldsymbol{r}_{i}\right)\right. \\
& \left.\times \frac{\boldsymbol{F}_{m_{1} m_{4}} \cdot \boldsymbol{F}_{m_{2} m_{3}}-3\left(\boldsymbol{F}_{m_{1} m_{4}} \cdot \boldsymbol{e}_{i j}\right)\left(\boldsymbol{F}_{m_{2} m_{3}} \cdot \boldsymbol{e}_{i j}\right)}{r_{i j}^{3}}\right\}
\end{aligned}
$$

In the above Hamiltonian we have used Einstein summation convention implying that repeated indices stand for summation. The first expression in the above equation 
represents the usual single body term where $\mu$ is chemical potential and $V_{\text {trap }}$ is the external trapping potential. The second expression stands for the short-range interaction between two atoms where $F$ is the hyperfine spin of each atom. The coupling constant $\mathcal{G}_{F}$, which corresponds to the scattering to a state with the total spin $F$, is given by $\mathcal{G}_{F}=4 \pi \hbar^{2} a_{S} / m$ with an $s$-wave scattering length $a_{F}$ and the mass of an atom $m$. Also, we set $\boldsymbol{e}_{i j}=\left(\boldsymbol{r}_{i}-\boldsymbol{r}_{j}\right) / r_{i j}$ and $r_{i j}=\left|\boldsymbol{r}_{i}-\boldsymbol{r}_{j}\right| . \mathcal{P}_{F}$ is the projection operator which projects the pair of "1" and "2" atoms into a state with total hyperfine spin $F$. The operator can be written using the Clebsch-Gordan coefficient $\left\langle m_{1} m_{2} \mid F M_{f}\right\rangle$;

$$
\left\langle m_{1} m_{2}\left|\mathcal{P}_{F}\right| m_{3} m_{4}\right\rangle=\sum_{M_{f}=-F}^{F}\left\langle m_{1} m_{2} \mid F M_{f}\right\rangle\left\langle F M_{f} \mid m_{3} m_{4}\right\rangle
$$

where $m_{i}$ is the spin sublevel of each atom, $F$ is the total spin of two atoms, $M_{f}$ is the spin sublevel of two atoms. The third term represents the long-ranged, anisotropic d-d interaction with $\boldsymbol{F}$ is given by $g_{F} \mu_{B} \boldsymbol{F}_{m_{i} m_{j}}=\left\langle m_{i}|\hat{\boldsymbol{\mu}}| m_{j}\right\rangle$. Here $\hat{\boldsymbol{\mu}}=-g_{S} \mu_{B} \hat{\boldsymbol{S}}+g_{I} \mu_{N} \hat{\boldsymbol{I}}, \mu_{N}$ is nuclear magneton, $\hat{\boldsymbol{S}}$ $(\hat{\boldsymbol{I}})$ is electronic (nuclear) spin angular momentum, and $g_{S, I}$ is Landé $g$ factors. Also, we set $c_{d d}=\mu_{0} g_{F}^{2} \mu_{B}^{2} /(4 \pi)$ with $\mu_{0}$ is magnetic permeability of the vacuum. Since the d-d interaction strength is proportional to $F^{2}$, it becomes more important for a large $F$ system. Moreover in such a system, $F+1$ contact interaction channels also exist. For these reasons, the analysis of such large $F$ system is very difficult and quite often hides the beauty of the quantum phase structures of such model under mathematical complexities.

Our purpose is to understand the effect of $d$-d interaction on rotationally invariant spin-textures. This requires us to understand the competition between rotationally invariant $s$-wave contact interaction and the anisotropic d-d interaction. To this purpose we have considered the case where all the interaction channels that represent the short range interaction except the one that accounts the $s$-wave repulsive interaction can be neglected in comparison to the d-d interaction. To motivate such a limit let us consider the case of the $F=1$ spinorial BEC $[8,9]$. Here the interaction is characterized by two scattering lengths $a_{0}$ and $a_{2}$, leading to the spin independent repulsive interaction $g \equiv g_{0}=\left(\mathcal{G}_{0}+2 \mathcal{G}_{2}\right) / 3=4 \pi \hbar^{2}\left(a_{0}+2 a_{2}\right) / 3 m$ in the triplet channel and the spin dependent exchange interaction $g_{2}=\left(\mathcal{G}_{2}-\mathcal{G}_{0}\right) / 3=4 \pi \hbar^{2}\left(a_{2}-a_{0}\right) / 3 m$ in the singlet channel. Since $a_{0}$ and $a_{2}$ are comparable in the typical experiments, $g_{2}$ is actually much smaller than $g_{0}$; $\left|g_{2}\right| / g \sim 1 / 10$ for ${ }^{23} \mathrm{Na}\left[25,26\right.$ ] and $\sim 1 / 35$ for ${ }^{87} \mathrm{Rb}[27$, 28.

This tendency that, except for the dominant repulsive part $g$, other spin-dependent channels are nearly cancelled, is likely to be correct for other F's [29] as well. Therefore for larger $F$, it is expected that the d-d interaction should become more important than these spin-spin interactions. For example, in Chromium $(F=3)$, it has been shown in reference [21. ( see the equation 3 of that paper) that the $s$-wave contact interaction in various spin channels is subdominant as compared to the dominant re- pulsive $s$-wave interaction. Where as the relative strength of the d-d interaction goes up due to higher value of the $F$ as well as $c_{d d}$ as well as $F$. Thus, to understand the interplay between the contact interaction and the dipole dipole interaction even for chromium BEC one can begin with only the dominant repulsive part of this short range interaction.

Once we accept this approximation, instead of working with $\boldsymbol{\Psi}(\mathbf{r})$ composed of the full quantum mechanical $2 F+1$ components $\left(\Psi_{F}, \Psi_{F-1}, \cdots, \Psi_{-F}\right)$, the order parameter can be simplified to $\boldsymbol{\Psi}\left(\mathbf{r}_{i}\right)=\psi\left(\mathbf{r}_{i}\right) \boldsymbol{S}\left(\mathbf{r}_{i}\right)$ where $\boldsymbol{S}\left(\mathbf{r}_{i}\right)$ is a classical vector with $\left|\boldsymbol{S}\left(\mathbf{r}_{i}\right)\right|^{2}=1$. Namely we can treat it as the classical spin vector whose magnitude $\left|\psi\left(\mathbf{r}_{i}\right)\right|^{2}$ is proportional to the local condensate density. As we see below such an approximation drastically simplify the Hamiltonian functional that represents such spinorial dipolar system while still yielding a rich variety of quantum phases that may observed in such systems as static configurations at various values of the system parameters. Naturally, larger the spin, better will be this approximation. Thus we investigate the stationary states of the system with large dipole moment using a classical spin approximation. Namely, our approximation is justified when $\left[\hat{s}_{i}, \hat{s}_{j}\right]=i \epsilon_{i, j, k} \hbar \hat{s}_{k} / S_{0} \ll 1$, where $\hat{s}_{i}$ is spin operator and $S_{0}$ is the magnitude of the spin. However, the current theory may also be applied to the spinor BECs with smaller value of spin, where our theory reproduces certain results similar to other studies [16]. This is very similar to the spin wave theory which should be valid for large spin only, but, works for spin $\frac{1}{2}$ particles as well.

At this point it is also appropriate to point out the limitations of such theory. For example, spin textures that arises out due to the effect of dipole dipole interaction on an anti ferromagnetic spin textures cannot be studied within this model since there is no term in the effective energy term ( without the d-d interaction) that favors the anti ferromagnetic ground state. We have completely neglect the terms that arises out of considering commutators of various spin operators as well. This condition is also exclude the possibility of observing the states which arises out of strong quantum fluctuations in spinor order parameter about it's mean field value. We shall point out some of the structures that appeared in the work of [16], [17, [21, 22, will not occur in this current theoretical framework.

The model Hamiltonian we propose is given by

$$
\begin{aligned}
H & =\int d^{3} \boldsymbol{r}_{i} \boldsymbol{\Psi}^{\dagger}\left(\boldsymbol{r}_{i}\right) H_{0}\left(\boldsymbol{r}_{i}\right) \Psi\left(\boldsymbol{r}_{i}\right) \\
& +\frac{g_{d}}{2} \iint d^{3} \boldsymbol{r}_{i} d^{3} \boldsymbol{r}_{j} V_{d d}\left(\boldsymbol{r}_{i}, \boldsymbol{r}_{j}\right)\left|\psi\left(\boldsymbol{r}_{i}\right)\right|^{2}\left|\psi\left(\boldsymbol{r}_{j}\right)\right|^{2},
\end{aligned}
$$

where

$$
\begin{aligned}
& H_{0}=-\frac{\hbar^{2}}{2 m} \nabla_{i}^{2}+V_{\text {trap }}\left(\boldsymbol{r}_{i}\right)-\mu+\frac{g}{2}\left|\boldsymbol{\Psi}\left(\boldsymbol{r}_{i}\right)\right|^{2}, \\
& V_{d d}\left(\boldsymbol{r}_{i}, \boldsymbol{r}_{j}\right)=\frac{\boldsymbol{S}_{i} \cdot \boldsymbol{S}_{j}-3\left(\boldsymbol{S}_{i} \cdot \boldsymbol{e}_{i j}\right)\left(\boldsymbol{S}_{j} \cdot \boldsymbol{e}_{i j}\right)}{r_{i j}^{3}} .
\end{aligned}
$$

The order parameter is represented by the spinor $\boldsymbol{\Psi}\left(\boldsymbol{r}_{i}\right)=$ $\psi\left(\boldsymbol{r}_{i}\right) \boldsymbol{S}_{i}$. The uniaxially symmetric trap potential is given 
by $V_{\text {trap }}(\boldsymbol{r})=\frac{1}{2} m \omega^{2}\left\{\gamma\left(x^{2}+y^{2}\right)+z^{2}\right\}$, where $\omega$ is the trap frequency in the transverse direction and $\gamma$ the anisotropy parameter. The short-range and d-d interaction parameters are introduced as $g$ and $g_{d}$, respectively. As explained earlier, the interactions that change each spin sublevel, are ignored since they are subdominant to the d-d interactions and only spin-independent short-range interactions are considered with coupling constant $g$. To implement the constraint $|\boldsymbol{S}|^{2}=1$ we parametrize it as :

$$
\boldsymbol{S}_{i} \equiv \boldsymbol{S}\left(\boldsymbol{r}_{i}\right)=\left[\begin{array}{c}
\cos \varphi\left(\boldsymbol{r}_{i}\right) \sin \theta\left(\boldsymbol{r}_{i}\right) \\
\sin \varphi\left(\boldsymbol{r}_{i}\right) \sin \theta\left(\boldsymbol{r}_{i}\right) \\
\cos \theta\left(\boldsymbol{r}_{i}\right)
\end{array}\right]
$$

The magnitude of the spin is included in the d-d interaction coupling constant $g_{d}$. The dimensionless form of this Hamiltonian functional of $\psi\left(\boldsymbol{r}_{i}\right), \theta\left(\boldsymbol{r}_{i}\right), \varphi\left(\boldsymbol{r}_{i}\right)$ is

$$
\begin{aligned}
H & =\frac{1}{2} \int d^{3} \boldsymbol{r}_{i}\left[\left|\nabla \psi\left(\boldsymbol{r}_{i}\right)\right|^{2}+n_{i}\left\{\left(\nabla \theta\left(\boldsymbol{r}_{i}\right)\right)^{2}\right.\right. \\
& \left.\left.+\sin ^{2} \theta\left(\boldsymbol{r}_{i}\right)\left(\nabla \varphi\left(\boldsymbol{r}_{i}\right)\right)^{2}+\gamma^{2}\left(x^{2}+y^{2}\right)+z^{2}\right\}-2 \mu n_{i}+g n_{i}^{2}\right] \\
& +\frac{g_{d}}{2} \iint d^{3} \boldsymbol{r}_{i} d^{3} \boldsymbol{r}_{j}\left(\frac{f\left(\boldsymbol{r}_{i}, \boldsymbol{r}_{j}\right)}{r_{i j}^{3}}-3 \frac{F\left(\boldsymbol{r}_{i}, \boldsymbol{r}_{j}\right)}{r_{i j}^{5}}\right) n_{i} n_{j}
\end{aligned}
$$

imaginary time $\tau$ :

$$
\begin{aligned}
& \frac{\partial \psi\left(\boldsymbol{r}_{i}, \tau\right)}{\partial \tau}=-\frac{\delta H}{\delta \psi^{*}\left(\boldsymbol{r}_{i}, \tau\right)} \\
& =\frac{1}{2} \nabla_{i}^{2} \psi_{i}+\left(\mu-g n_{i}\right) \psi_{i} \\
& \quad-\frac{1}{2}\left\{\left(\nabla_{i} \theta_{i}\right)^{2}+\sin ^{2} \theta_{i}\left(\nabla_{i} \varphi_{i}\right)^{2}+\gamma^{2}\left(x_{i}^{2}+y_{i}^{2}\right)+z_{i}^{2}\right\} \psi_{i} \\
& \quad-g_{d} \int d \boldsymbol{r}_{j}\left(\frac{f\left(\boldsymbol{r}_{i}, \boldsymbol{r}_{j}\right)}{r_{i j}^{3}}-3 \frac{F\left(\boldsymbol{r}_{i}, \boldsymbol{r}_{j}\right)}{r_{i j}^{5}}\right) n_{j} \psi_{i}, \\
& \frac{\partial \theta}{}\left(\boldsymbol{r}_{i}, \tau\right) \\
& \quad \partial \tau=-\frac{\delta H}{\delta \theta\left(\boldsymbol{r}_{i}, \tau\right)} \\
& \quad\left(\nabla_{i}^{2} \theta_{i}\right) n_{i}+\left(\nabla_{i} \theta_{i}\right) \cdot\left(\nabla_{i} n_{i}\right)-\sin \theta_{i} \cos \theta_{i}\left(\nabla_{i} \varphi_{i}\right)^{2} n_{i} \\
& \quad-g_{d} \int d \boldsymbol{r}_{j}\left\{\cos \left(\varphi_{j}-\varphi_{i}\right) \sin \theta_{j} \cos \theta_{i}-\sin \theta_{i} \cos \theta_{j}\right\} \frac{n_{i} n_{j}}{r_{i j}^{3}} \\
& \quad+3 g_{d} \int d \boldsymbol{r}_{j}\left\{x_{i j}^{2} \sin \theta_{j} \cos \theta_{i} \cos \varphi_{i} \cos \varphi_{j}\right. \\
& \quad+y_{i j}^{2} \sin \theta_{j} \cos \theta_{i} \sin \varphi_{i} \sin \varphi_{j}-z_{i j}^{2} \sin \theta_{i} \cos \theta_{j} \\
& \quad+x_{i j} y_{i j} \sin \theta_{j} \cos \theta_{i} \sin \left(\varphi_{i}+\varphi_{j}\right) \\
& \quad+y_{i j} z_{i j}\left(\cos \theta_{j} \cos \theta_{i} \sin \varphi_{i}-\sin \theta_{j} \sin \theta_{i} \sin \varphi_{j}\right) \\
& \left.\left.\quad+z_{i j} x_{i j}\left(\cos \theta_{j} \cos \theta_{i} \cos \varphi_{i}-\sin \theta_{j} \sin \theta_{i} \cos \varphi_{j}\right)\right\} \frac{n_{i} n_{j}}{r_{i j}^{5}} 13\right)
\end{aligned}
$$

and

where

$$
\begin{aligned}
& f\left(\boldsymbol{r}_{i}, \boldsymbol{r}_{j}\right)=\cos \left(\varphi_{i}-\varphi_{j}\right) \sin \theta_{i} \sin \theta_{j}+\cos \theta_{i} \theta_{j}, \\
& F\left(\boldsymbol{r}_{i}, \boldsymbol{r}_{j}\right)=x_{i j}^{2} \cos \varphi_{i} \cos \varphi_{j} \sin \theta_{i} \sin \theta_{j} \\
& \quad+y_{i j}^{2} \sin \varphi_{i} \sin \varphi_{j} \sin \theta_{i} \sin \theta_{j}+z_{i j}^{2} \cos \theta_{i} \cos \theta_{j} \\
& \quad+2 x_{i j} y_{i j} \sin \left(\varphi_{i}+\varphi_{j}\right) \sin \theta_{i} \sin \theta_{j}+2 y_{i j} z_{i j} \sin \varphi_{i} \sin \theta_{i} \cos \theta_{j} \\
& \quad+2 z_{i j} x_{i j} \cos \varphi_{i} \sin \theta_{i} \cos \theta_{j},
\end{aligned}
$$

with $\left|\psi\left(\boldsymbol{r}_{i}\right)\right|^{2}=\left|\psi_{i}\right|^{2}=n_{i}, \theta_{i}=\theta\left(\boldsymbol{r}_{i}\right), \varphi_{i}=\varphi\left(\boldsymbol{r}_{i}\right)$, and $\alpha_{i j}=\alpha\left(\boldsymbol{r}_{i}\right)-\alpha\left(\boldsymbol{r}_{j}\right)(\alpha=x, y, z)$. We note that the spin gradient term in the first line in Eq. (9) represents the nonlinear sigma model [1,30] with the corresponding spin stiffness is given by superfluid density. Thus the gradient energy term gives the leading order coupling between the spin with mass density and leads to a number of interesting phenomenon in such condensates. Higher order coupling between spin and density comes from the d-d interaction. The d-d interaction represents interaction between the different parts of the spin density. Even though the rotational invariance of the NLSM is lost due to the explicit appearance of angular fields $\phi$ and $\theta$ in the energy density, the system still have reflection symmetry in the $x y, y z$ and $z x$ plane. In the subsequent analysis we shall see that this reflection symmetry is manifested in the equilibrium configurations of the condensates. The energy (length) is scaled by the harmonic frequency $\omega$ (harmonic length $d=1 / \sqrt{m \omega}$ ) with $\hbar=1$. The functional derivatives with respect to $\psi\left(\boldsymbol{r}_{i}\right)^{*}, \theta\left(\boldsymbol{r}_{i}\right)$, and $\varphi\left(\boldsymbol{r}_{i}\right)$ lead to the corresponding the time-dependent GP equations in the

$$
\begin{aligned}
& \frac{\partial \varphi\left(\boldsymbol{r}_{i}, \tau\right)}{\partial \tau}=-\frac{\delta H}{\delta \varphi\left(\boldsymbol{r}_{i}, \tau\right)} \\
& =\sin ^{2} \theta_{i}\left(\nabla_{i}^{2} \varphi_{i}\right)+2 \sin \theta_{i} \cos \theta_{i}\left(\nabla_{i} \theta_{i}\right) \cdot\left(\nabla_{i} \varphi_{i}\right) n_{i} \\
& \quad+\sin ^{2} \theta_{i}\left(\nabla_{i} \varphi_{i}\right) \cdot\left(\nabla_{i} n_{i}\right) \\
& \quad-g_{d} \int d \boldsymbol{r}_{j} \sin \left(\varphi_{j}-\varphi_{i}\right) \sin \theta_{j} \sin \theta_{i} \frac{n_{i} n_{j}}{r_{i j}^{3}} \\
& \quad+3 g_{d} \int d \boldsymbol{r}_{j}\left\{-x_{i j}^{2} \sin \theta_{j} \sin \theta_{i} \sin \varphi_{i} \cos \varphi_{j}\right. \\
& \quad+y_{i j}^{2} \sin \theta_{j} \sin \theta_{i} \sin \varphi_{j} \cos \varphi_{i} \\
& \quad+x_{i j} y_{i j} \sin \theta_{j} \sin \theta_{i} \cos \left(\varphi_{i}+\varphi_{j}\right) \\
& \quad+y_{i j} z_{i j} \sin \theta_{i} \cos \theta_{j} \cos \varphi_{i} \\
& \left.\quad-z_{i j} x_{i j} \cos \theta_{j} \sin \theta_{i} \cos \varphi_{i}\right\} \frac{n_{i} n_{j}}{r_{i j}^{5}} .
\end{aligned}
$$

These equations describe the time evolution of order parameters for a "imaginary time" $\tau$, which rolls along the slope of the energy functionals. For $\tau \rightarrow \infty$, the order parameters converge to the stationary configuration, corresponding to one of the local minima of the energy functional. We numerically solved the set of the GP equations (12)-(14) in imaginary time $(\tau=i t)$.In this paper, for a fixed repulsive interaction $\left(g / \omega d^{3}=0.01\right)$, we vary the d-d interaction $g_{d}$ in a range of $0 \leq g_{d} \leq 0.4 g$, beyond which the system is unstable. We consider two types of the confinement to see the effect of of the trap anisotropy on the d-d interaction: namely a cigar $(\gamma=5.0)$ and a pancake $(\gamma=0.2)$ trap . The total particle number is kept around $N \sim 10^{4}$. The three-dimensional space is discretized into the lattice sites $\sim 2.5 \times 10^{4}$. 


\section{STABLE CONFIGURATIONS}

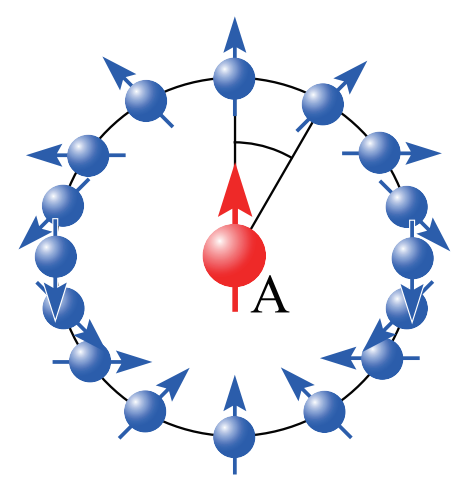

Fig. 1. color online: Anisotropy of the d-d interaction considering two spins. The spins around the spin A at the center are pointing the direction of the d-d energy minimum. The top and bottom spins are likely to be parnell to the spin A or head-totail texture, and the spins at the side of spin A are likely to be antiparallel to the spin A.

First, let us consider the anisotropic nature of the d$\mathrm{d}$ interaction. Figure 1 shows the anisotropy of the d-d interaction with the help of two spins (one at the center and the another is taken around it). The directions of the surrounding correspond to the energy minimum. Those spins at the top and the bottom are likely to be parallel to the spin A or head-to-tail texture, and the spins at both sides of spin A are anti-parallel to the spin A. Now we shall describe a number of equilibrium spin-density configuration that are obtained by minimizing the energy expression (9). In Fig. 2 (a) and Fig. 3 (a) we have respectively plotted the spin-profile of the z-flare structure as well as its density profile in the $x z$ plane which is obtained in a cigar shaped trap $(\gamma=5)$. This spin texture is the typical example of the dipolar effect on an otherwise ferromagnetic structure, that is the ground state of isotropic NLSM. At the trap center, where the density is the highest, we can have a spin vector (dipole moment) pointing toward $z$ direction. And the other spins placed right and left are laid in the head-to-tail texture. The spins near the surface of the system are bent as those in Fig. 1 . As $g_{d}$ increases $(=0.1 g$ and $0.2 g)$, the bending angle of the flare spin texture increases and the system gets elongated along the $z$ direction. The typical length scale over the change of the spin angle takes place is determined by the relative strength of the gradient energy term to the term representing the $\mathrm{d}-\mathrm{d}$ interaction. At the center, the spin-stiffness or the superfluid density is higher and hence bending the spin is energetically costly. Thus the energy will be minimized by reducing the gradient energy. At the edge the spin-stiffness is lower and and hence the bending of the spin will cost less gradient energy. The texture in Fig. 2(c) is another example that shows strong coupling of the density and the spin texture and it also breaks the axial symmetry of the density in the $x-y$ plane. The texture is the so-called "r-flare" texture, and is one of the (a)

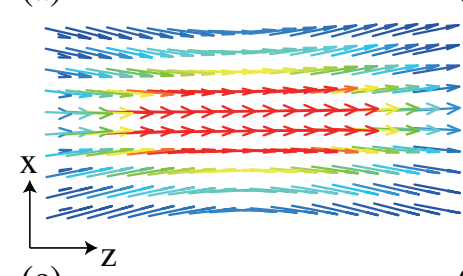

(c)

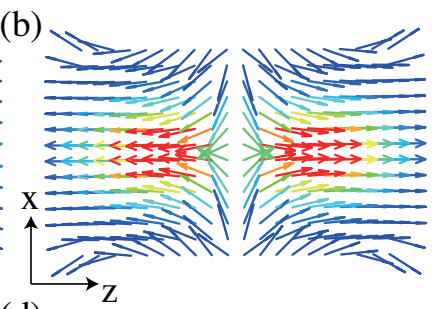

(d)
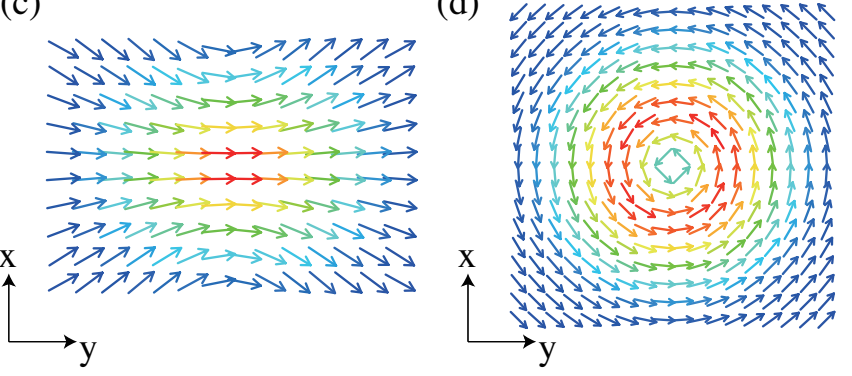

Fig. 2. color online: Spin texture $\boldsymbol{S}(\boldsymbol{r})$ of the stationary states. Four figures are correspond to (a) z-flare texture, (b) two-zflare texture, (c) r-flare texture, and (d) spin current texture. (a) and (b) are in the cigar type trap potential $\gamma=5$. (c) and (d) are in the pancake type trap potential $\gamma=0.2$. The $\mathrm{d}-\mathrm{d}$ interaction strength are all the same value $g_{d}=0.2 g$. The color is corresponds to the local density.

minimum energy configurations in a disk-shaped pancake geometry, namely when $\gamma \ll 1$. Because of the stronger confinement along the $z$-axis the gradient energy cost will be higher in that direction. Therefore the flaring will now take place in $x-y$ plane, rather than in the $x-z$ plane. Let us assume, the spins are aligned to one direction in the $x$ $y$ plane (e.g., the $x$ direction in Fig. 2(c). Fig. 3(c) shows the cross-section density plots of the particle number (the corresponding spin texture). The spins are almost parallel to the $x$ axis, but they bend away at the outer region. At the $x$ - $y$ plane around $z=0$, the condensate gets elongated to the direction along the spin polarization. This is because of the d-d interaction, which favors the headto-tail arrangement, and the particle number is increased at the center. The bending angle of the spins on the circumference increases with increasing $g_{d}$ in the same way it happens in the case of $z$-flare configuration. The two structure that has been discussed above are non-singular in terms of density as well as the spin-profile even though they have shown strong intertwining between the spin and density. We shall now discuss two cases where this intertwining lead to singularity in either density or spin-profile or in the both. The spin and density profile of one of such equilibrium structure is shown in Fig. 2(b) and Fig 3 (b). This configuration is obtained from the initial condition of a hedgehog texture like topological defect in which all the spins point outward from the origin, i.e., $\mathbf{S}(\boldsymbol{r})=\boldsymbol{r} /|\boldsymbol{r}|$. The solution presented here can also be regarded as the two "z-flare" textures stacked back to back. Here, the density at the trap center is decreased to avoid spin gradient energy cost even though this region represents the minimum of the trap potential and finally leads to the splitting of the condensate. This clearly demonstrates the strong- 
coupling between spin and density. The leading order contribution comes from the spin gradient energy whose coefficient or stiffness is proportional to the density of the condensate. But the dipole-dipole interaction term also contributes to this process since it includes all the higher order derivatives of the spinorial fields. The final configuration which we shall discuss in this context is the socalled "spin current" texture. This structure combines a vortex in the density field with a circulation current in the spin-texture. Thus this serves as an another typical configuration in which the spin texture and the particle density are strongly coupled through the d-d interaction. Symmetrically on the both side of the density depleted region, the spins are locally aligned to head-to-tail texture and the whereas spins are antiparallel (see Fig. 2(d)). This texture is explained by the anisotropy of the d-d interaction depicted in Fig. 1. At the trap center, the density depletion is occurred over the coherent length $\xi_{d}$ of the d-d interaction which is shown in Fig. 3(d). In the present case, we find $\xi_{d} \sim 2.0 \xi_{c}$ ( $\xi_{c}$ is the ordinary coherent length of the repulsive interaction). This is because to avoid spin gradient energy cost. For an alternative explanation of the spin current texture we rewrite the d-d interaction in Eq. (9) as $V_{d d}\left(\boldsymbol{r}_{i j}\right) \propto\left(g_{d} / r_{i j}^{3}\right) \sum_{\mu=-1}^{2} Y_{2 \mu}(\cos \theta) \sum_{\mu}(i j)$ with $\sum_{\mu}(i j)$ being a rank 2 tensor consisting of the two spins at $i$ and $j$ sites, and $Y_{2 \mu}(\cos \theta)$ a spherical harmonics 31. $\theta$ is the polar angle in spherical coordinates of the system. The spin current texture shown in Fig. 2(d) picks up the phase factor $e^{2 i \phi}$ when winding around the origin. This is coupled to $Y_{2 \pm 2}(\cos \theta) \propto \sin ^{2} \theta$, meaning that this orbital moment dictates the number density depletion at the pancake center. The spin-orbit coupling directly manifests itself here. The total angular momentum consisting of the spin and orbit ones is a conserved quantity of the present axis-symmetric system, leading to the Einstein-de Haas effect 16. The spin current texture is stable for the wide range of anisotropy $\gamma: 0.01 \leq \gamma \leq 0.6$, beyond which it becomes unstable. Some of the spin textures are similar to those obtained by Kawaguchi et al. [16] for $F=1$ case, such as the flare (the flower in their terminology) and the spin current textures (the polar core vortex). The latter texture demonstrates the interaction between the orbital and spin angular momenta, ultimately leading to the Einstein-de Haas effect mentioned in connection with $F=3{ }^{52}$ Cr case [13] and $F=1$ case [16].

\section{STABILITY REGIME FOR VARIOUS QUANTUM PHASES}

In the previous section 3 we have described how energy minimization process in presence of dipole-dipole interaction takes advantage of the strong coupling between mass and spin-density and stabilizes a number of topologically non trivial structure. All these structures correspond to various quantum mechanical ground states of the corresponding many body systems. The transition of the system from one macroscopic configuration to another as a function of various external parameters such as the cou- (a)

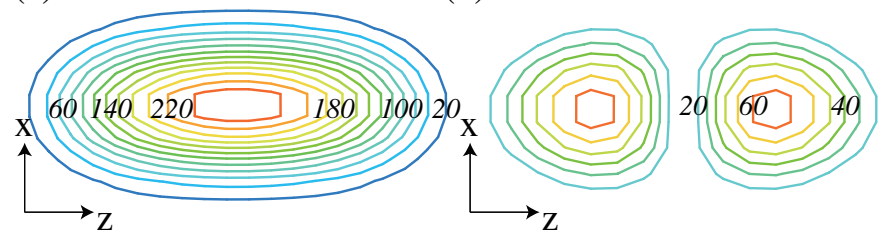

(c)

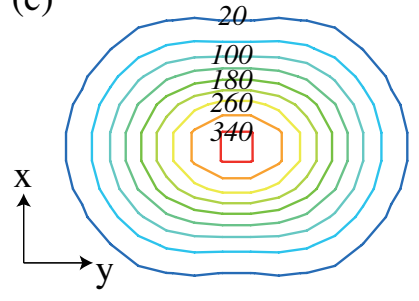

(d)

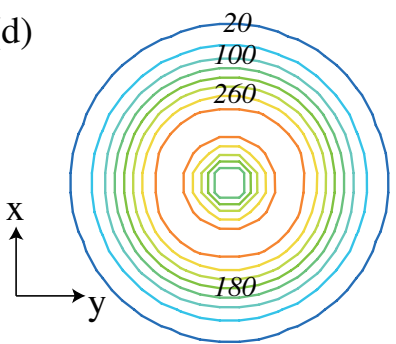

Fig. 3. color online Density profile of the stationary states. Four figures are correspond to (a) z-flare texture, (b) two-zflare texture, (c) r-flare texture, and (d) spin current texture. (a) and (b) are in the cigar type trap potential $\gamma=5$. (c) and (d) are in the pancake type trap potential $\gamma=0.2$. The $\mathrm{d}-\mathrm{d}$ interaction strength are all the same value $g_{d}=0.2 \mathrm{~g}$. In the spin current texture (d), the density is torus structure. The maximum point of the density are not at the center in (b) and (d), due to their spin structures.

pling constant $\frac{g_{d}}{g}$ that gives the relative strength of dipolar interaction, anisotropy of the trap $\gamma$, and the chemical potential $\frac{E}{N}$, actually implies quantum phase transition when applied to infinite system. Indeed the system on which we perform our numerical computation is finite and results are also limited by the numerical accuracy. Moreover we only have been able to focus on some limited data points in the three dimensional phase space of external parameters as described above. Nevertheless based on this we can provide some indication of interesting quantum phase transitions or cross-over in the actual system. We make a few comments in this direction and then summarize our numerical results in a tabular form. For example the rflare and the spin-current texture both are stabilized in a pancake geometry. To see what external condition energetically prefers one above the other, we have looked at the how the energy $E$ varies as a function of the total number of particles for a given aspect ratio is $\gamma=0.2$ and coupling strength $g_{d}=0.2 g$. We found that in the small $N$ region, the r-flare texture is favorable, but in the larger $N$ region, the spin current texture has the lower energy. The z-flare texture is never the ground state even though sometimes it corresponds to a local energy minima at these values of the parameters. These can be explained as follows: At the small $N$ region, the system size is comparable with the coherence length $\xi_{d}$ of the d-d interaction. Thus it costs more spin gradient energy than those in large $N$. The zflare texture can not be the lowest energy state because the alignment and elongation to $z$ direction cost the higher energy than those to the radial direction at this value of the trap anisotropy (aspect ratio). The $z$-flare spin texture is however stable for $g_{d}<0.3 g$ and for $N \sim 2.3 \times 10^{4}$, and stay robust for various aspect ratios such as: $\gamma=0.2$ and 
Please give a shorter version with: \authorrunning and \titlerunning prior to \maketitle

Table 1. Energy of various stable configurations.

\begin{tabular}{|c|c|c|c|}
\hline$\gamma$ & $\frac{g_{d}}{g}$ & $\frac{E}{N}$ & type \\
\hline 0.05 & 0.20 & 1.535 & $\mathbf{s c}$ \\
\hline 0.20 & 0.10 & 1.855 & $\mathbf{z f}$ \\
\hline 0.20 & 0.10 & 2.716 & $\mathbf{z f}$ \\
\hline 0.20 & 0.10 & 1.617 & rf \\
\hline 0.20 & 0.10 & 2.386 & rf \\
\hline 0.20 & 0.10 & 1.569 & $\mathbf{s c}$ \\
\hline 0.20 & 0.10 & 2.344 & $\mathbf{s c}$ \\
\hline 0.20 & 0.20 & 2.308 & $\mathbf{z f}$ \\
\hline 0.20 & 0.20 & 1.900 & rf \\
\hline 0.20 & 0.20 & 1.988 & $\mathbf{s c}$ \\
\hline 5.00 & 0.29 & 1.800 & $\mathbf{z f}$ \\
\hline
\end{tabular}

5.0, but it corresponds to the minimum energy configuration only in a cigar shaped trap $\gamma \gg 1$. Again, in this regime $r$-flare structure is stable, but has higher energy compared to the z-flare structure. This also points out the another aspect of this way of computing the ground state energy. The stable solutions may correspond to one of the local minima of the energy landscape and thus a comparison between the energies corresponding to various such local minima is necessary to understand the actual equilibrium structure. As we increase the coupling strength beyond $g_{d}=0.4 \mathrm{~g}$ for the particle number of order $10^{4}$, none of the above mentioned structure is stable and due to attractive part of the $\mathrm{d}$-d interaction the condensate collapse. However this value of $g_{d}$ is typically higher than the critical interaction strength where all dipoles are polarized in a given direction. In the following paragraph we put some of these results in a tabular form. Here rf means r-flare, zf means z flare, and sc means spin-current texture. The last column not necessarily indicate the ground state, but only an equilibrium structure within computational accuracy. For example comparing the data in 8th, 9th, and 10th row of the table which are for the almost same number of particles we find that the rf structure is the energetically most favored structure. Similarly a comparison between the 8th and 11th row indicates that as one goes from a pancake to a cigar shaped trap the $\mathbf{z f}$ structure becomes energetically becomes more favorable.

\section{Theory of collective excitations}

The next interesting issue will be to study the nature of collective excitations around such ground state configurations. The methodology is similar to the study of collective excitations in ordinary BEC, however now for these spinorial order parameter which represents strong intertwining between the density and the spin degrees of freedom. We can therefore expect that some of these resulting collective excitations strong coupling between density waves such as phonons with various types of spin waves. The method is outlined here briefly. Our order parameter consists of three field, namely

$$
\chi=\left[\begin{array}{l}
\chi_{1} \\
\chi_{2} \\
\chi_{3}
\end{array}\right]=\left[\begin{array}{l}
\psi \\
\theta \\
\varphi
\end{array}\right]
$$

As GP solution, $\psi$ can be complex whereas the angular fields $\theta$ and $\varphi$ are real. We consider the small fluctuations around each of this field of the form

$$
\chi_{i} \Rightarrow \chi_{i}+\lambda\left(u_{i} e^{i \omega t}-v_{i} e^{-i \omega t}\right)
$$

For $\left\{v_{i}\right\}$ we take the complex conjugate. We then subsequently inserts them in the field equations and in the spirit of linear response theory write the resulting equations by collecting the terms that appears as coefficients of $\lambda$. We neglect all other terms which appears with higher power of $\lambda$. These equations are very long because of the presence of the $d-d$ interaction in the mean field energy functional. We therefore include the $d-d$ interaction term only symbolically and illustrate them in one case explicitly

The equations for the collective excitations are respectively:

For $\psi$

$$
\begin{aligned}
\omega u_{1}= & \frac{1}{2}\left[\nabla^{2}+2 \mu-4 g|\psi|^{2}-(\nabla \theta)^{2}-\sin ^{2} \theta(\nabla \varphi)^{2}-V_{\text {trap }}\right] u_{1} \\
& -\frac{1}{2} \psi\left[(\nabla \theta) \nabla+\sin 2 \theta(\nabla \varphi)^{2}\right] u_{2}-\frac{1}{2} \psi \sin ^{2} \theta(\nabla \varphi) \nabla u_{3} \\
& +g \psi^{2} v_{1}+\frac{1}{2} \psi(\nabla \theta) \nabla v_{2}+\frac{1}{2} \psi \sin ^{2} \theta(\nabla \varphi) \nabla v_{3} \\
& +\mathrm{d}-\mathrm{d}_{\text {contrib }} \\
\omega v_{1}= & -\frac{1}{2}\left[\nabla^{2}+2 \mu-4 g|\psi|^{2}-(\nabla \theta)^{2}-\sin ^{2} \theta(\nabla \varphi)^{2}-V_{\text {trap }}\right] v_{1} \\
& +\frac{1}{2} \psi^{*}\left[(\nabla \theta) \nabla+\sin 2 \theta(\nabla \varphi)^{2}\right] v_{2}+\frac{1}{2} \psi^{*} \sin ^{2} \theta(\nabla \varphi) \nabla v_{3} \\
& -g\left(\psi^{*}\right)^{2} u_{1}-\frac{1}{2} \psi(\nabla \theta) \nabla u_{2}+\frac{1}{2} \psi^{*} \sin ^{2} \theta(\nabla \varphi) \nabla u_{3} \\
& +\mathrm{d}-\mathrm{d}_{\text {contrib }}
\end{aligned}
$$

For $\theta$

$$
\begin{aligned}
\omega u_{2}= & {\left[\psi^{*}\left(\nabla^{2} \theta\right)+(\nabla \theta)\left(\left(\nabla \psi^{*}\right)+\psi^{*} \nabla\right)-\frac{1}{2} \sin 2 \theta(\nabla \varphi)^{2} \psi^{*}\right] u_{1} } \\
& +\left[|\psi|^{2} \nabla^{2}+\left(\nabla|\psi|^{2}\right) \nabla-\frac{1}{2} \cos 2 \theta(\nabla \varphi)^{2}|\psi|^{2}\right] u_{2} \\
& -\frac{1}{2} \sin 2 \theta(\nabla \phi)|\psi|^{2} \nabla u_{3} \\
& -\left[\left(\nabla^{2} \theta\right) \psi+(\nabla \theta)((\nabla \psi)+\psi \nabla)-\frac{1}{2} \sin 2 \theta(\nabla \varphi)^{2} \psi\right] v_{1} \\
& +\sin \theta \cos \varphi|\psi|^{2}(\nabla \varphi) \nabla v_{3}+\text { d-d } \mathrm{d}_{\text {contrib }}
\end{aligned}
$$




$$
\begin{aligned}
\omega v_{2}= & \left.-\left[\psi\left(\nabla^{2} \theta\right)+(\nabla \theta)((\nabla \psi)+\psi \nabla)\right)-\frac{1}{2} \sin 2 \theta(\nabla \varphi)^{2} \psi\right] v_{1} \\
& -\left[|\psi|^{2} \nabla^{2}+\left(\nabla|\psi|^{2}\right) \nabla-\frac{1}{2} \cos 2 \theta(\nabla \varphi)^{2}|\psi|^{2}\right] v_{2} \\
& +\frac{1}{2} \sin 2 \theta(\nabla \phi)|\psi|^{2} \nabla v_{3} \\
& +\left[\left(\nabla^{2} \theta\right) \psi+(\nabla \theta)\left(\left(\nabla \psi^{*}\right)+\psi^{*} \nabla\right)-\frac{1}{2} \sin 2 \theta|\nabla \varphi|^{2} \psi\right] u_{1} \\
& +\sin \theta \cos \varphi|\psi|^{2}(\nabla \varphi) \nabla u_{3}+\mathrm{d}-\mathrm{d}_{\text {contrib }}
\end{aligned}
$$

For $\phi$

$$
\begin{aligned}
\omega u_{3}= & {\left[\sin 2 \theta(\nabla \phi)(\nabla \theta) \psi^{*}+\sin ^{2} \theta(\nabla \varphi)\left(\left(\nabla \psi^{*}\right)+\psi^{*} \nabla\right)\right] u_{1} } \\
& +\left[\frac { 1 } { 2 } \operatorname { s i n } 2 \theta \left[\left(\nabla^{2} \varphi\right)+2(\nabla \varphi)\left[|\psi|^{2} \nabla+\left(\nabla|\psi|^{2}\right)\right]\right.\right. \\
& \left.+\cos 2 \theta(\nabla \theta)(\nabla \phi)|\psi|^{2}\right] u_{2} \\
& +\left[\sin ^{2} \theta\left\{|\psi|^{2} \nabla^{2}+\left(\nabla|\psi|^{2}\right) \nabla\right\}+\sin 2 \theta(\nabla \theta)|\psi|^{2} \nabla\right] u_{3} \\
& -\left[\sin 2 \theta(\nabla \theta)(\nabla \varphi) \psi+\sin ^{2} \theta(\nabla \varphi)((\nabla \psi)+\psi \nabla)\right] v_{1} \\
& +\mathrm{d}-\mathrm{d}_{\text {contrib }}
\end{aligned}
$$

$$
\begin{aligned}
\omega v_{3}= & -\left[\sin 2 \theta(\nabla \phi)(\nabla \theta) \psi+\sin ^{2} \theta(\nabla \varphi)((\nabla \psi)+\psi \nabla)\right] v_{1} \\
& -\left[\frac { 1 } { 2 } \operatorname { s i n } 2 \theta \left[\left(\nabla^{2} \varphi\right)+2(\nabla \varphi)\left[|\psi|^{2} \nabla+\left(\nabla|\psi|^{2}\right)\right]\right.\right. \\
& \left.-\cos 2 \theta(\nabla \theta)(\nabla \phi)|\psi|^{2}\right] v_{2} \\
& -\left[\sin ^{2} \theta\left\{|\psi|^{2} \nabla^{2}+\left(\nabla|\psi|^{2}\right) \nabla\right\}+\sin 2 \theta(\nabla \theta)|\psi|^{2} \nabla\right] v_{3} \\
& +\left[\sin 2 \theta(\nabla \theta)(\nabla \varphi) \psi^{*}+\sin ^{2} \theta(\nabla \varphi)\left(\left(\nabla \psi^{*}\right)+\psi^{*} \nabla\right)\right] u_{1} \\
& +\mathrm{d}-\mathrm{d}_{\text {contrib }}
\end{aligned}
$$

These gives us a set of six equations for six $u_{i} \mathrm{~s}$ and $v_{i} \mathrm{~s}$. Their solutions describe the nature of the collective excitations. The $d-d$ interaction contribution to the first equations for this set (17) (after collecting the coefficients of $e^{i \omega t} \mathrm{~S}$ only) will be for example

$$
\begin{aligned}
\mathrm{d}-\mathrm{d}_{\text {contrib }}= & -g_{d} \int d \boldsymbol{r}_{\boldsymbol{i}}\left(\frac{f_{i j}}{r_{i j}^{3}}-3 \frac{F_{i j}}{r_{i j}^{5}}\right) \psi_{j}\left(\psi_{i}^{*} u_{1 i}+\psi_{i} v_{1 i}\right) \\
& -g_{d} \int d r_{i}\left(\frac{f_{i j}}{r_{i j}^{3}}-3 \frac{F_{i j}}{r_{i j}^{5}}\right) n_{i} u_{1 j} \\
& -g_{d} \int d r_{i} \mathcal{F}_{i j} n_{i} \psi_{j}
\end{aligned}
$$

where

$$
\begin{aligned}
\mathcal{F}_{i j}= & \frac{1}{r_{i j}^{3}}\left[\left\{\cos \left(\phi_{i}-\phi_{j}\right) \cos \theta_{i} \sin \theta_{j}-\sin \theta_{i} \cos \theta_{j}\right\} u_{2 i}\right. \\
& +\left\{\cos \left(\phi_{i}-\phi_{j}\right) \sin \theta_{i} \cos \theta_{j}-\cos \theta_{i} \sin \theta_{j}\right\} u_{2 j} \\
& \left.-\sin \left(\phi_{i}-\phi_{j}\right) \sin \theta_{i} \sin \theta_{j}\left(u_{3 i}-u_{3 j}\right)\right] \\
& -3 \frac{1}{r_{i j}^{5}}\left[\left\{x_{i j}^{2} \cos \theta_{i} \sin \theta_{j} \cos \phi_{i} \cos \phi_{j}\right.\right. \\
& +y_{i j}^{2} \cos \theta_{i} \sin \theta_{j} \sin \phi_{i} \sin \phi_{j} \\
& -z_{i j}^{2} \sin \theta_{i} \cos \theta_{j} \\
& +x_{i j} y_{i j} \cos \theta_{i} \sin \theta_{j} \sin \left(\phi_{i}+\phi_{j}\right) \\
& +2 y_{i j} z_{i j} \cos \theta_{i} \cos \theta_{j} \sin \phi_{i} \\
& \left.-2 z_{i j} x_{i j} \sin \theta_{i} \sin \theta_{j} \cos \phi_{i}\right\} u_{2 i} \\
& +\left\{x_{i j}^{2} \sin \theta_{i} \cos \theta_{j} \cos \phi_{i} \cos \phi_{j}\right. \\
& +y_{i j}^{2} \sin \theta_{i} \cos \theta_{j} \sin \phi_{i} \sin \phi_{j} \\
& -z_{i j}^{2} \cos \theta_{i} \sin \theta_{j} \\
& +x_{i j} y_{i j} \sin \theta_{i} \cos \theta_{j} \sin \left(\phi_{i}+\phi_{j}\right) \\
& -2 y_{i j} z_{i j} \sin \theta_{i} \sin \theta_{j} \sin \phi_{i} \\
& \left.+2 z_{i j} x_{i j} \cos \theta_{i} \cos \theta_{j} \cos \phi_{i}\right\} u_{2 j} \\
& +\left\{-x_{i j}^{2} \sin \theta_{i} \sin \theta_{j} \sin \phi_{i} \cos \phi_{j}\right. \\
& +y_{i j}^{2} \sin \theta_{i} \sin \theta_{j} \cos \phi_{i} \cos \phi_{j} \\
& +x_{i j} y_{i j} \sin \theta_{i} \sin \theta_{j} \cos \left(\phi_{i}+\phi_{j}\right) \\
& +2 y_{i j} z_{i j} \sin \theta_{i} \cos \theta_{j} \cos \phi_{i} \\
& \left.-2 z i j x_{i j} \cos \theta_{i} \sin \theta j \sin \phi_{i}\right\} u_{3 i} \\
& +\left\{-x_{i j}^{2} \sin \theta_{i} \sin \theta_{j} \cos \phi_{i} \sin \phi_{j}\right. \\
& +y_{i j}^{2} \sin \theta_{i} \sin \theta_{j} \sin \phi_{i} \cos \phi_{j} \\
& \left.\left.+x_{i j} y_{i j} \sin \theta_{i} \sin \theta_{i} \sin \theta_{j} \cos \left(\phi_{i}+\phi_{j}\right)\right\} u_{3 j}\right] \quad(24)
\end{aligned}
$$

In the similar manner all other contributions can also be written Full numerical solutions of these equations will be submitted for publication in a later communication. Here we therefore made some comments by pointing out the general nature of the collective excitations under the applications of a weak uniform field. The simplest limit is the case without dipolar interaction and the trapping poferro

The above set of equations become

$$
\begin{aligned}
\omega u_{1} & =\left[\frac{1}{2} \nabla^{2}+\mu-2 g|\psi|^{2}\right] u_{1}+g|\psi|^{2} v_{1} \\
\omega v_{1} & =-\left[\frac{1}{2} \nabla^{2}+\mu-2 g|\psi|^{2}\right] v_{1}-g|\psi|^{2} u_{1} \\
\omega u_{2} & =|\psi|^{2} \nabla^{2} u_{2} \\
\omega v_{2} & =-|\psi|^{2} \nabla^{2} v_{2} \\
\omega u_{3} & =|\psi|^{2} \sin ^{2} \theta \nabla^{2} u_{3} \\
\omega v_{3} & =-|\psi|^{2} \sin ^{2} \theta \nabla^{2} v_{3}
\end{aligned}
$$

The first set of equations (25) describes the usual Bogoliubov modes for the uniform condensate with

$$
\omega= \pm \sqrt{\varepsilon_{k}\left(\varepsilon_{k}+2 g|\psi|^{2}\right)}, \varepsilon_{k}=\frac{k^{2}}{2}
$$


where we have used $\mu=g|\psi|^{2}$ for the uniform condensate. In the long wavelength limit this gives

$$
\omega \propto k, k \ll 1
$$

the usual phonon mode. The two other equations (26) and (27) gives

$$
\omega \propto k^{2}
$$

which gives transverse spin wave modes which are gapless. The spin stiffness is proportional to superfluid density. Thus the density and spin are strongly coupled. Also the longitudinal spin mode cannot appear in the system since the length of the spins are fixed in our model and thus such elastic vibrations are forbidden. These various collective modes will determine the nature of the stability of the quantum phases that we have described in our work. One can also make some qualitative comments general nature of some collective oscillations above various ground state configurations. For example the collective oscillations over the spin current texture will intertwine the collective modes of vortices with higher winding number with spin waves and it will be very interesting to study these modes.

\section{Comparison with other methods}

A natural question that arises how our method can be compared with the other methods,particularly described in references [21,22] where the full quantum mechanical nature of the spin for Chromium BEC has been taken into account. A detailed comparison is not possible due to different treatment of the dipolar and short range interaction in both these works and the type of order parameters that has been considered in reference [22. However following issues can be pointed out. In eq. 3 of reference 21 the interaction strength of the short range interaction in various channels has been provided. It has been shown clearly there that all the spin dependent channels for the short range interaction is sub dominant as compared to rotationally invariant contact interaction in the maximum spin channel $\left(c_{0}\right.$ in 21]). Thus the basic assumption of our theoretical formulation agrees with the analysis of this work. A major difference also comes from the single mode approximation adopted in that work, where as in our work the spin can rotate continuously as the field $\theta$ and $\phi$ varies. This is why stabilization of spin current texture like state is more natural in our method while there in 21] one needs to go beyond SMA to observe such structures. Additionally they have also assumed a polarized dipolar condensate as the equilibrium structure. This is not the case in our analysis particularly for spin current texture as well as the hedgehog structure. Another difference in the methodology is that in the analysis of [21] varying $\frac{g_{0}}{g_{6}}($ these are equivalent to interaction strengths in the singlet and triplet channel for spin-1 bosons) certain phases has been obtained. We have completely neglected the effect of singlet channel. Due to this the phases that can be stabilized by long wavelength fluctuations around the ferromagnetic phase can be observed in our analysis. However the phases which can be observed due to the condensation of spin-flip type of excitations around the ferromagnetic phases cannot be captured within our model. A possible extension of our model can be done by explicitly breaking the spin rotational symmetry that will allow such spin singlet excitations.

A comparison with the model proposed by Ho et al. is even more difficult since the order parameter considered there is very different. However given the general agreement between the phase diagram obtained in ref. 21] and 22. the differences between our findings and those of reference 22] can be traced back to the same reasons we have just mentioned.

\section{SUMMARY AND OUTLOOK}

In this paper we have reported the calculation of a a number of interesting equilibrium configuration of the spinor dipolar Bose-Einstein condensates treating the dipole as a classical spin and neglecting all other s-wave type interactions in various spin channels except the Hatree type repulsive one. The resulting equilibrium configurations shows strong coupling between mass and spin density, indications of quantum phase transitions and the presence of topological singularities in density and spin fields. These spin textures can be observed directly via a novel phasesensitive in situ detection [4 or indirectly via conventional absorption imaging for the number density. Interesting magnetometric measurement is also possible to detect such spin-textures [19]. For the future work, it is interesting to study vortex state, especially spin current texture. Since the density is already depleted for the spin current texture, such a vortex can be easily observable and might become stable. We have also provided the theoretical framework in which the collective excitation spectrum can be studied. In such excitations spin and density waves will get strongly coupled. These are still difficult problem because of long-ranged anisotropic nature of the d-d interaction. Moreover, the model Hamiltonian is applicable literally for electric dipolar systems without further approximation. We expect that BECs of hetero-nuclear molecules with permanent electric dipole moment might be realized in near future [32] where the formation of textures presented here may be possible. Lastly, the above model being an effective theory it also has limitations. For example, the structures that can be attributed to the other spin-dependent channel of the s-wave interaction, cannot be obtained within this method. Including these effects in this description would be highly interesting theoretical problem. Nevertheless the hallmark of this model is that it unfurls a number of topologically non trivial quantum phases and explain their existence in a very transparent method .

\section{ACKNOWLEDGEMENTS}

We thank Tarun K. Ghosh and W. Pogosov for useful discussions in the early stage of this research. This work 
was supported by a grant of the Japan Society for the Promotion of Science. One of us (SG) thanks IIT Delhi to provide financial support to attend the Grenoble BEC 2008 conference where this work has been presented as a poster.

\section{References}

1. A. A. Belavin and A. M. Polyakov, Pis'ma Zh. Eksp. Teor. Fiz. 22, 503 (1975) [SPIRES]; [JETP Lett. 22, 245 (1975)];

2. R. Rajaraman, Solitons and Instantons (North-Holland, Amsterdam, 1989)

3. U. A. Khawaja and H. C. Stoof, Nature 411, 918 (2001); J. Ruostekosky and J. R. Anglin, Phys. Rev. Lett, 86, 3934 (2001); R. A. Battye, N. R. Cooper and P. M. Sutcliffe, Phys. Rev. Lett, 88, 080401 (2002).

4. See for example, L. E. Sadler, J. M. Higbie, S. R. Leslie, M. Vengalattore, and D. M. Stamper-Kurn, Nature (London) 443, 312 (2006)

5. S. L. Sondhi, A. Karlhede, S. A. Kivelson, and E. H. Rezayi, Phys. Rev. B 47, 16419 (1993)

6. K. Moon, H. Mori, Kun Yang, S.M. Girvin, A.H. MacDonald, L. Zheng, D. Yashioka, and Shou-Cheng Zhang, Phys. Rev. B 51, 5138 (1995).

7. S. Ghosh and R. Rajaraman, Phys. Rev. B 63, 035304 (2001).

8. T. Ohmi and K. Machida, J. Phys. Soc. Jpn. 67, 1822 (1998).

9. T. -L. Ho, Phys. Rev. Lett. 81, 742 (1998).

10. D.M. Stamper-Kurn, M.R. Andrews, A.P. Chikkatur, S. Inouye, H.-J. Miesner, J. Stenger, and W. Ketterle, Phys. Rev. Lett., 80, 2027 (1998)

11. A. Görlitz, T.L. Gustavson, A.E. Leanhardt, R. LoNw, A.P. Chikkatur, S. Gupta, S. Inouye, D.E. Pritchard, and W. Ketterle, Phys. Rev. Lett, 90, 090401, (2003)

12. A. Griesmaier et al., Phys. Rev. Lett. 94, 160401 (2005).

13. J. Stuhleret al., Phys. Rev. Lett. 95, 150406 (2005); A. Griesmaier et al., Phys. Rev. Lett. 97, 250402 (2006); S. Giovanazzi et al., Phys. Rev. A 74, 013621 (2005).

14. Thierry Lahaye et al. Nature, 448, 672 (2007).

15. See for review, M. A. Baranov et al., Phys. Scr. T 102, 74 (2002).

16. Y. Kawaguchi et al., Phys. Rev. Lett. 96, 080405 (2006); 97, 130404 (2006); and 98, 110406 (2007).

17. S. Yi and H. Pu, Phys. Rev. Lett. 97, 020401 (2006).

18. R. Cheng et al., J. Phys. B 38, 2569 (2005).

19. M. Vengalattore, S. R. Leslie, J. Guzman, and D. M. Stamper-Kurn, Phys. Rev. Lett., 100, 170403 (2008).

20. Austen Lamacraft, Phys. Rev. A 77, 063622 (2008)

21. L. Santos and T. Pfau, Phys. Rev. Lett. 96, 190404 (2006);

22. R. B. Diener and T. -L. Ho, Phys. Rev. Lett. 96, 190405 (2006).

23. M. Takahashi, S. Ghosh, T. Mizushima, and K. Machida, Phys. Rev. Lett. 98, 260403 (2007).

24. L. Fadeev and A. Niemi, Nature 387, 58 (1997).

25. J. Stenger, S. Inouye, D. M. Stamper-Kurn, H. -J. Miesner, A. P. Chikkatur, and W. Ketterle, Nature (London) 396, 345 (1998).

26. J. P. Burke, C. H. Greene, and J. L. Bohn, Phys. Rev. Lett. 81, 3355 (1998).

27. M. D. Barrett et al., Phys. Rev. Lett. 87, 010404 (2001).
28. N. N. Klausen, J. L. Bohn, and C. H. Greene, Phys. Rev. A 64, 053602 (2001).

29. For ${ }^{87} \mathrm{Rb}(F=2)$, the two spin dependent interactions are 80 and 50 times smaller than the spin-independent one. T. Kuwamoto et al., Phys. Rev. A 69, 063604 (2004).

30. F.D.M. Haldane, Phys. Rev. Lett. 50, 1153(1983).

31. C. J. Pethick and H. Smith, in Bose-Einstein condensation in dilute gases (Cambridge University Press, Cambridge, 2002). Chap. 5, (5.76).

32. See the special issue on ultracold polar molecules; Eur. Phys. J. D31, 149-445 (2004). 